\title{
BASIC PROPERTIES OF SOBOLEV'S SPACES ON TIME SCALES
}

\author{
RAVI P. AGARWAL, VICTORIA OTERO-ESPINAR, KANISHKA PERERA, \\ AND DOLORES R. VIVERO
}

Received 18 January 2006; Accepted 22 January 2006

We study the theory of Sobolev's spaces of functions defined on a closed subinterval of an arbitrary time scale endowed with the Lebesgue $\Delta$-measure; analogous properties to that valid for Sobolev's spaces of functions defined on an arbitrary open interval of the real numbers are derived.

Copyright (c) 2006 Ravi P. Agarwal et al. This is an open access article distributed under the Creative Commons Attribution License, which permits unrestricted use, distribution, and reproduction in any medium, provided the original work is properly cited.

\section{Introduction}

Sobolev's spaces are a fundamental tool in real analysis, for instance, in the use of variational methods to solve boundary value problems in ordinary and partial differential equations and difference equations. In spite of this, theory for functions defined on an arbitrary bounded open interval of the real numbers is well known, see [2], and for functions defined on an arbitrary bounded subset of the natural numbers is trivial, as far as we know, for functions defined on an arbitrary time scale, it has not been studied before.

The aim of this paper is to give an introduction to Sobolev's spaces of functions defined on a closed interval $[a, b] \cap \mathbb{T}$ of an arbitrary time scale $\mathbb{T}$ endowed with the Lebesgue $\Delta$ measure. In Section 2, we gather together the concepts one needs to read this paper, such as the $L^{p}$ spaces linked to the Lebesgue $\Delta$-measure and absolutely continuous functions on an arbitrary closed interval of $\mathbb{T}$. The most important part of this paper is Section 3 where we define the first-order Sobolev's spaces as the space of $L_{\Delta}^{p}([a, b) \cap \mathbb{T})$ functions whose generalized $\Delta$-derivative belongs to $L_{\Delta}^{p}([a, b) \cap \mathbb{T})$, moreover, we study some of their properties by establishing an equivalence between them and the usual Sobolev's spaces defined on an open interval of the real numbers. Section 4 is devoted to the generalization of Sobolev's spaces to order $n \geq 2$.

\section{Preliminaries}

The Lebesgue $\Delta$-measure $\mu_{\Delta}$ was defined in [1, Section 5.7] or in [5, Section 5] as the Carathéodory extension of a set function and it may be characterized in terms of 
well-known measures as the following result shows; we refer the reader to [6-8] for a broad introduction to measure and integration theory.

Proposition 2.1. The Lebesgue $\Delta$-measure is defined over the Lebesgue measurable subsets of $\mathbb{\mathbb { T }}$; moreover, it satisfies the following equality:

$$
\mu_{\Delta}= \begin{cases}\lambda+\sum_{i \in I}\left(\sigma\left(t_{i}\right)-t_{i}\right) \cdot \delta_{t_{i}}+\mu_{M}, & \text { if } M \in \mathbb{T}, \\ \lambda+\sum_{i \in I}\left(\sigma\left(t_{i}\right)-t_{i}\right) \cdot \delta_{t_{i}}, & \text { if } M \notin \mathbb{T},\end{cases}
$$

where $\left\{t_{i}\right\}_{i \in I}, I \subset \mathbb{N}$, is the set of all right-scattered points of $\mathbb{T}, M$ is the supremum of $\mathbb{T}, \lambda$ is the Lebesgue measure, $\delta_{t_{i}}$ is the Dirac measure concentrate at $t_{i}$, and $\mu_{M}$ is a degenerate measure defined as $\mu_{M}(A)=0$ if $M \notin A$ and $\mu_{M}(A)=+\infty$ if $M \in A$.

Proof. From properties of measure, one can deduce relation (2.1) for the outer measures linked to these measures which plainly yields to (2.1).

As a straightforward consequence of equality (2.1), one can deduce the following formula to calculate the Lebesgue $\Delta$-integral; this formula was proved in [4], nevertheless, we remark that this argument is more simple than that.

Proposition 2.2. Let $E \subset \mathbb{T}$ be a $\Delta$-measurable set. If $f: \mathbb{T} \rightarrow \mathbb{R}$ is $\Delta$-integrable on $E$, then

$$
\int_{E} f(s) \Delta s=\int_{E} f(s) d s+\sum_{i \in I_{E}}\left(\sigma\left(t_{i}\right)-t_{i}\right) \cdot f\left(t_{i}\right)+r(f, E),
$$

where

$$
r(f, E)= \begin{cases}\mu_{M}(E) \cdot f(M), & \text { if } M \in \mathbb{T}, \\ 0, & \text { if } M \notin \mathbb{T},\end{cases}
$$

$I_{E}:=\left\{i \in I: t_{i} \in E\right\}$ and $\left\{t_{i}\right\}_{i \in I}, I \subset \mathbb{N}$, is the set of all right-scattered points of $\mathbb{T}$.

Definition 2.3. Let $A \subset \mathbb{T}$. $A$ is called $\Delta$-null set if $\mu_{\Delta}(A)=0$. Say that a property $P$ holds $\Delta$-almost everywhere ( $\Delta$-a.e.) on $A$, or for $\Delta$-almost all ( $\Delta$-a.a.) $t \in A$ if there is a $\Delta$-null set $E \subset A$ such that $P$ holds for all $t \in A \backslash E$.

Definition 2.4. Let $E \subset \mathbb{T}$ be a $\Delta$-measurable set and let $p \in \overline{\mathbb{R}} \equiv[-\infty,+\infty]$ be such that $p \geq 1$ and let $f: E \rightarrow \mathbb{R}$ be a $\Delta$-measurable function. Say that $f$ belongs to $L_{\Delta}^{p}(E)$ provided that either

$$
\int_{E}|f|^{p}(s) \Delta s<\infty \quad \text { if } p \in \mathbb{R}
$$

or there exists a constant $C \in \mathbb{R}$ such that

$$
|f| \leq C \quad \Delta \text {-a.e. on } E \quad \text { if } p=+\infty \text {. }
$$

Note that equality (2.2) guarantees that in order for $f: \mathbb{T} \rightarrow \mathbb{R}$ to belong to $L_{\Delta}^{p}(\mathbb{T})$, $p \in \mathbb{R}$, and $\mathbb{T}$ bounded from above, it is necessary that $f(M)=0$. We will work with the 
$L_{\Delta}^{p}\left(J^{o}\right)$ spaces, where $J=[a, b] \cap \mathbb{T}, a, b \in \mathbb{T}, a<b$, is an arbitrary closed subinterval of $\mathbb{T}$ and $J^{o}=[a, b) \cap \mathbb{T}$; we state some of their properties whose proofs can be found in [6-8].

Theorem 2.5. Let $p \in \overline{\mathbb{R}}$ be such that $p \geq 1$. Then, the set $L_{\Delta}^{p}\left(J^{o}\right)$ is a Banach space together with the norm defined for every $f \in L_{\Delta}^{p}\left(J^{o}\right)$ as

$$
\|f\|_{L_{\Delta}^{p}}:= \begin{cases}{\left[\int_{J^{o}}|f|^{p}(s) \Delta s\right]^{1 / p},} & \text { if } p \in \mathbb{R}, \\ \inf \left\{C \in \mathbb{R}:|f| \leq C \Delta \text {-a.e. on } J^{o}\right\}, & \text { if } p=+\infty .\end{cases}
$$

Moreover, $L_{\Delta}^{2}\left(J^{o}\right)$ is a Hilbert space together with the inner product given for every $(f, g) \in$ $L_{\Delta}^{2}\left(J^{o}\right) \times L_{\Delta}^{2}\left(J^{o}\right)$ by

$$
(f, g)_{L_{\Delta}^{2}}:=\int_{J^{o}} f(s) \cdot g(s) \Delta s
$$

Proposition 2.6. Suppose $p \in \overline{\mathbb{R}}$ and $p \geq 1$. Let $p^{\prime} \in \overline{\mathbb{R}}$ be such that $1 / p+1 / p^{\prime}=1$.

Then, if $f \in L_{\Delta}^{p}\left(J^{o}\right)$ and $g \in L_{\Delta}^{p^{\prime}}\left(J^{o}\right)$, then $f \cdot g \in L_{\Delta}^{1}\left(J^{o}\right)$ and

$$
\|f \cdot g\|_{L_{\Delta}^{1}} \leq\|f\|_{L_{\Delta}^{p}} \cdot\|g\|_{L_{\Delta}^{p^{\prime}}} .
$$

This expression is called Hölder's inequality and Cauchy-Schwarz's inequality whenever $p=2$.

Proposition 2.7. If $p \in \mathbb{R}$ and $p \geq 1$, then, the set $C_{c}\left(J^{o}\right)$ of all continuous functions on $J^{o}$ with compact support in $J^{o}$ is dense in $L_{\Delta}^{p}\left(J^{o}\right)$.

As a consequence of Proposition 2.2, one can establish the following equivalence between the $L_{\Delta}^{p}\left(J^{o}\right)$ spaces and the usual $L^{p}([a, b])$ spaces linked to the Lebesgue measure.

Corollary 2.8. Let $p \in \overline{\mathbb{R}}$ with $p \geq 1$, let $f: J \rightarrow \overline{\mathbb{R}}$, and let $\tilde{f}:[a, b] \rightarrow \overline{\mathbb{R}}$ be the extension of $f$ to $[a, b]$ defined as

$$
\tilde{f}(t):= \begin{cases}f(t), & \text { if } t \in J, \\ f\left(t_{i}\right), & \text { if } t \in\left(t_{i}, \sigma\left(t_{i}\right)\right), \text { for some } i \in I_{J},\end{cases}
$$

with $I_{J}:=\left\{i \in I: t_{i} \in J\right\}$ and $\left\{t_{i}\right\}_{i \in I}, I \subset \mathbb{N}$, is the set of all right-scattered points of $\mathbb{T}$.

Then, $f \in L_{\Delta}^{p}\left(J^{o}\right)$ if and only if $\tilde{f} \in L^{p}([a, b])$. In this case,

$$
\|f\|_{L_{\Delta}^{p}}=\|\tilde{f}\|_{L^{p}}
$$

As we know from general theory of Sobolev's spaces, another important class of functions is just the absolutely continuous functions.

Definition 2.9. A function $f: J \rightarrow \mathbb{R}$ is said to be absolutely continuous on $J, f \in A C(J)$, if for every $\varepsilon>0$, there exists a $\delta>0$ such that if $\left\{\left[a_{k}, b_{k}\right) \cap \mathbb{T}\right\}_{k=1}^{n}$, with $a_{k}, b_{k} \in J$, is a finite pairwise disjoint family of subintervals of $J$ satisfying $\sum_{k=1}^{n}\left(b_{k}-a_{k}\right)<\delta$, then $\sum_{k=1}^{n}\left|f\left(b_{k}\right)-f\left(a_{k}\right)\right|<\varepsilon$. 
4 Basic properties of Sobolev's spaces on time scales

These functions are precisely that for which the fundamental theorem of Calculus holds.

Theorem 2.10 [3, Theorem 4.1]. A function $f: J \rightarrow \mathbb{R}$ is absolutely continuous on $J$ if and only if $f$ is $\Delta$-differentiable $\Delta$-a.e. on $J^{o}, f^{\Delta} \in L_{\Delta}^{1}\left(J^{o}\right)$ and

$$
f(t)=f(a)+\int_{[a, t) \cap \mathbb{\pi}} f^{\Delta}(s) \Delta s, \quad \forall t \in J .
$$

Absolutely continuous functions on $\mathbb{T}$ verify the integration by parts formula.

THeorem 2.11. If $f, g: J \rightarrow \mathbb{R}$ are absolutely continuous functions on $J$, then $f \cdot g$ is absolutely continuous on $J$ and the following equality is valid:

$$
\int_{J^{o}}\left(f^{\Delta} g+f^{\sigma} g^{\Delta}\right)(s) \Delta s=f(b) g(b)-f(a) g(a)=\int_{J^{o}}\left(f g^{\Delta}+f^{\Delta} g^{\sigma}\right)(s) \Delta s .
$$

They are linked to the class of absolutely continuous functions on $[a, b]$ as the following property shows.

Corollary $2.12[3$, Corollary 3.1]. Assume that $f: J \rightarrow \mathbb{R}$ and define $\bar{f}:[a, b] \rightarrow \mathbb{R}$ as

$$
\bar{f}(t):= \begin{cases}f(t), & \text { if } t \in J, \\ f\left(t_{i}\right)+\frac{f\left(\sigma\left(t_{i}\right)\right)-f\left(t_{i}\right)}{\sigma\left(t_{i}\right)-t_{i}}\left(t-t_{i}\right), & \text { if } t \in\left(t_{i}, \sigma\left(t_{i}\right)\right), \text { for some } i \in I_{J},\end{cases}
$$

with $I_{J}:=\left\{i \in I: t_{i} \in J\right\}$ and $\left\{t_{i}\right\}_{i \in I}, I \subset \mathbb{N}$, is the set of all right-scattered points of $\mathbb{T}$.

Then, $f$ is absolutely continuous on $J$ if and only if $\bar{f}$ is absolutely continuous on $[a, b]$.

Moreover, for every $n \in \mathbb{N}, n \geq 1$, we will denote as

$$
A C^{n}(J):=\left\{x \in A C(J): x^{\Delta^{j}} \in A C\left(J^{k^{j}}\right) \forall j \in\{1, \ldots, n\}\right\},
$$

where for every $j \in \mathbb{N}, j \geq 1, J^{\kappa^{j}}=\left[a, \rho^{j}(b)\right] \cap \mathbb{T}$.

\section{First-order Sobolev's spaces}

The aim of this section is to study the first-order Sobolev's spaces on $J$ equipped with the Lebesgue $\Delta$-measure.

Definition 3.1. Let $p \in \overline{\mathbb{R}}$ be such that $p \geq 1$ and $u: J \rightarrow \overline{\mathbb{R}}$. Say that $u$ belongs to $W_{\Delta}^{1, p}(J)$ if and only if $u \in L_{\Delta}^{p}\left(J^{o}\right)$ and there exists $g: J^{\kappa} \rightarrow \mathbb{\mathbb { R }}$ such that $g \in L_{\Delta}^{p}\left(J^{o}\right)$ and

$$
\int_{J^{o}}\left(u \cdot \varphi^{\Delta}\right)(s) \Delta s=-\int_{J^{o}}\left(g \cdot \varphi^{\sigma}\right)(s) \Delta s \quad \forall \varphi \in C_{0, r d}^{1}\left(J^{\kappa}\right)
$$

with

$$
C_{0, r d}^{1}\left(J^{\kappa}\right):=\left\{f: J \longrightarrow \mathbb{R}: f \in C_{r d}^{1}\left(J^{\kappa}\right), f(a)=0=f(b)\right\}
$$

and $C_{r d}^{1}\left(J^{\kappa}\right)$ is the set of all continuous functions on $J$ such that they are $\Delta$-differentiable on $J^{\kappa}$ and their $\Delta$-derivatives are $r d$-continuous on $J^{\kappa}$. 
The integration by parts formula for absolutely continuous functions on $J$ establishes that the relation

$$
V_{\Delta}^{1, p}(J):=\left\{x \in A C(J): x^{\Delta} \in L_{\Delta}^{p}\left(J^{o}\right)\right\} \subset W_{\Delta}^{1, p}(J)
$$

is true for every $p \in \overline{\mathbb{R}}$ with $p \geq 1$. We will show that both sets are, as class of functions, equivalent; for this purpose, we need the following lemmas.

Lemma 3.2. Let $f \in L_{\Delta}^{1}\left(J^{o}\right)$ be such that the following equality is true:

$$
\int_{J^{o}}(f \cdot u)(s) \Delta s=0, \quad \forall u \in C_{c}\left(J^{o}\right)
$$

then

$$
f \equiv 0 \quad \Delta \text {-a.e. on } J^{o} .
$$

Proof. Fix $\varepsilon>0$, the density of $C_{c}\left(J^{o}\right)$ in $L_{\Delta}^{1}\left(J^{o}\right)$ guarantees the existence of $f_{1} \in C_{c}\left(J^{o}\right)$ such that $\left\|f-f_{1}\right\|_{L_{\Delta}^{1}}<\varepsilon$, and so, by (3.4), we deduce that for every $u \in C_{c}\left(J^{o}\right)$, it is true that

$$
\left|\int_{J^{o}}\left(f_{1} \cdot u\right)(s) \Delta s\right| \leq\|u\|_{C\left(J^{o}\right)} \cdot\left\|f-f_{1}\right\|_{L_{\Delta}^{1}}<\varepsilon\|u\|_{C\left(J^{o}\right)} .
$$

Because the sets

$$
A_{1}:=\left\{s \in J^{o}: f_{1}(s) \geq \varepsilon\right\}, \quad A_{2}:=\left\{s \in J^{o}: f_{1}(s) \leq-\varepsilon\right\}
$$

are compact and disjoint subsets of $J^{o}$, Urysohn's lemma allows to construct a function $u_{0}: J^{o} \rightarrow \mathbb{R}$ which belongs to $C_{c}\left(J^{o}\right)$ and it verifies

$$
u_{0} \equiv\left\{\begin{array}{ll}
1 ; & \text { on } A_{1}, \\
-1 ; & \text { on } A_{2},
\end{array} \quad\left|u_{0}\right| \leq 1 \text { on } J^{o} ;\right.
$$

so that, by defining $A:=A_{1} \cup A_{2}$, we have that

$$
\begin{aligned}
\int_{J^{o}}\left|f_{1}\right|(s) \Delta s= & \int_{J^{o}}\left(f_{1} \cdot u_{0}\right)(s) \Delta s-\int_{J^{o} \backslash A}\left(f_{1} \cdot u_{0}\right)(s) \Delta s \\
& +\int_{J^{o} \backslash A}\left|f_{1}\right|(s) \Delta s \leq \varepsilon+2 \varepsilon(b-a) .
\end{aligned}
$$

As a consequence of the arbitrary choice of $\varepsilon>0$, we achieve (3.5).

Lemma 3.3. Let $f \in L_{\Delta}^{1}\left(J^{o}\right)$. Then, a necessary and sufficient condition for the validity of the equality

$$
\int_{J^{o}}\left(f \cdot \varphi^{\Delta}\right)(s) \Delta s=0, \quad \text { for every } \varphi \in C_{0, r d}^{1}\left(J^{\mathcal{K}}\right),
$$

is the existence of a constant $c \in \mathbb{R}$ such that

$$
f \equiv c \quad \Delta \text {-a.e. on } J^{o} .
$$


Proof. The necessary condition is consequence of the fundamental theorem of Calculus. Conversely, fix $u \in C_{c}\left(J^{o}\right)$ arbitrary; by defining $h, \varphi: J \rightarrow \mathbb{R}$ as

$$
\begin{aligned}
h(t): & = \begin{cases}u(t)-\frac{\int_{J^{o}} u(r) \Delta r}{b-a}, & \text { if } t \in J^{o}, \\
-\frac{\int_{J^{o}} u(r) \Delta r}{b-a}, & \text { if } t=b,\end{cases} \\
\varphi(t): & =\int_{[a, t) \cap \mathbb{\pi}} h(s) \Delta s, \quad \forall t \in J,
\end{aligned}
$$

the fundamental theorem of Calculus establishes that $\varphi \in C_{0, r d}^{1}\left(J^{\kappa}\right)$ and so, equality (3.10) yields to

$$
\begin{aligned}
0 & =\int_{J^{o}}\left[f \cdot\left(u-\frac{\int_{J^{o}} u(r) \Delta r}{b-a}\right)\right](s) \Delta s \\
& =\int_{J^{o}}\left[\left(f-\frac{\int_{J^{o}} f(r) \Delta r}{b-a}\right) \cdot u\right](s) \Delta s .
\end{aligned}
$$

Therefore, Lemma 3.2 allows to deduce (3.11) with $c=\int_{J^{\circ}} f(r) \Delta r /(b-a)$.

Now, we are able to prove the characterization of functions in $W_{\Delta}^{1, p}(J)$ in terms of functions in $V_{\Delta}^{1, p}(J)$.

Theorem 3.4. Suppose that $u \in W_{\Delta}^{1, p}(J)$ for some $p \in \overline{\mathbb{R}}$ with $p \geq 1$ and that (3.1) holds for $g \in L_{\Delta}^{p}\left(J^{o}\right)$. Then, there exists a unique function $x \in V_{\Delta}^{1, p}(J)$ such that the equalities

$$
x=u, \quad x^{\Delta}=g \quad \Delta \text {-a.e. on } J^{o}
$$

are satisfied.

Moreover, if $g \in C_{r d}\left(J^{\kappa}\right)$, then there exists a unique function $x \in C_{r d}^{1}\left(J^{\kappa}\right)$ such that

$$
x=u \quad \Delta \text {-a.e. on } J^{o}, \quad x^{\Delta}=g \quad \text { on } J^{\kappa} .
$$

Proof. Define $v: J \rightarrow \mathbb{R}$ as

$$
v(t):=\int_{[a, t) \cap \mathbb{\pi}} g(s) \Delta s, \quad \forall t \in J
$$

the fundamental theorem of Calculus guarantees that $v \in V_{\Delta}^{1, p}(J)$ and by the integration by parts formula, we have that for every $\varphi \in C_{0, r d}^{1}\left(J^{\kappa}\right)$,

$$
\int_{J^{o}}\left[(v-u) \cdot \varphi^{\Delta}\right](s) \Delta s=-\int_{J^{o}}\left[\left(v^{\Delta}-g\right) \cdot \varphi^{\sigma}\right](s) \Delta s=0
$$

so that, Lemma 3.3 ensures the existence of a constant $c \in \mathbb{R}$ such that $v-u \equiv c \Delta$-almost everywhere on $J^{\circ}$. As a consequence of the fundamental theorem of Calculus we conclude that function $x: J \rightarrow \mathbb{R}$ defined as $x(t):=v(t)-c$ for all $t \in J$ is the unique function in $V_{\Delta}^{1, p}(J)$ for which (3.14) is valid. 
Furthermore, if $g \in C_{r d}\left(J^{\kappa}\right)$, then the fundamental theorem of Calculus establishes that $x \in C_{r d}^{1}\left(J^{\kappa}\right)$ and $x^{\Delta}=g$ on $J^{\kappa}$.

By identifying every function in $W_{\Delta}^{1, p}(J)$ with its absolutely continuous representative in $V_{\Delta}^{1, p}(J)$ for which (3.14) holds, the set $W_{\Delta}^{1, p}(J)$ can be endowed with the structure of Banach space.

Theorem 3.5. Assume $p \in \overline{\mathbb{R}}$ and $p \geq 1$. The set $W_{\Delta}^{1, p}(J)$ is a Banach space together with the norm defined for every $x \in W_{\Delta}^{1, p}(J)$ as

$$
\|x\|_{W_{\Delta}^{1, p}}:=\|x\|_{L_{\Delta}^{p}}+\left\|x^{\Delta}\right\|_{L_{\Delta}^{p}} .
$$

Moreover, the set $H_{\Delta}^{1}(J):=W_{\Delta}^{1,2}(J)$ is a Hilbert space together with the inner product given for every $(x, y) \in H_{\Delta}^{1}(J) \times H_{\Delta}^{1}(J)$ by

$$
(x, y)_{H_{\Delta}^{1}}:=(x, y)_{L_{\Delta}^{2}}+\left(x^{\Delta}, y^{\Delta}\right)_{L_{\Delta}^{2}} .
$$

Proof. Let $\left\{x_{n}\right\}_{n \in \mathbb{N}}$ be a Cauchy sequence in $W_{\Delta}^{1, p}(J)$; Theorem 2.5 guarantees the existence of $u, g \in L_{\Delta}^{p}\left(J^{o}\right)$ such that $\left\{x_{n}\right\}_{n \in \mathbb{N}}$ and $\left\{x_{n}^{\Delta}\right\}_{n \in \mathbb{N}}$ converge strongly in $L_{\Delta}^{p}\left(J^{o}\right)$ to $u$ and $g$, respectively, and so, by taking limits in the equality

$$
\int_{J^{o}}\left(x_{n} \cdot \varphi^{\Delta}\right)(s) \Delta s=-\int_{J^{o}}\left(x_{n}^{\Delta} \cdot \varphi^{\sigma}\right)(s) \Delta s, \quad \varphi \in C_{0, r d}^{1}\left(J^{\kappa}\right),
$$

we conclude that $u \in W_{\Delta}^{1, p}(J)$. Thereby, it follows from Theorem 3.4, that there exists $x \in W_{\Delta}^{1, p}(J)$ such that $\left\{x_{n}\right\}_{n \in \mathbb{N}}$ converges strongly in $W_{\Delta}^{1, p}(J)$ to $x$.

3.1. Some properties. We will derive some properties of the Banach space $W_{\Delta}^{1, p}(J)$; the first one asserts that $W_{\Delta}^{1, p}(J)$ is continuously inmersed into $C(J)$ equipped with the supremum norm $\|\cdot\|_{C(J)}$.

Proposition 3.6. Assume $p \in \overline{\mathbb{R}}$ with $p \geq 1$, then there exists a constant $K>0$, only dependent on $b-a$, such that the inequality

$$
\|x\|_{C(J)} \leq K \cdot\|x\|_{W_{\Delta}^{1, p}}
$$

holds for all $x \in W_{\Delta}^{1, p}(J)$ and hence, the immersion $W_{\Delta}^{1, p}(J) \hookrightarrow C(J)$ is continuous.

Proof. Fix $x \in W_{\Delta}^{1, p}(J)$. Let $t, T \in J$ be such that $|x(t)|:=\min _{s \in \mathbb{T}}|x(s)|$ and $|x(T)|:=$ $\max _{s \in \mathbb{T}}|x(s)|$; there is no harm in assuming $t \leq T$. The fundamental theorem of Calculus and Hölder's inequality lead to

$$
\|x\|_{C(J)} \leq|x(t)|+\int_{[t, T) \cap \mathbb{T}}\left|x^{\Delta}\right|(s) \Delta s \leq K \cdot\|x\|_{W_{\Delta}^{1, p}}
$$

for some $K>0$, only dependent on $b-a$.

The strong compactness criterion in $C(J)$ and Proposition 3.6 allow to prove the following compactness property in $C(J)$. 
Proposition 3.7. Let $p \in \overline{\mathbb{R}}$ be such that $p \geq 1$. Then, the following statements are true.

(1) If $p>1$, then the immersion $W_{\Delta}^{1, p}(J) \hookrightarrow C(J)$ is compact.

(2) If $p=1$, then the immersion $W_{\Delta}^{1, p}(J) \hookrightarrow C(J)$ is compact if and only if every point of $J$ is isolated.

Proof. Denote by $\mathscr{F}_{p} p$ the closed unit ball in $W_{\Delta}^{1, p}(J)$; we know from Theorem 3.4 that $\mathscr{F} p$ is closed and bounded in $C(J)$.

If $p>1$, then the fundamental theorem of Calculus and Hölder's inequality ensure that $\mathscr{F}_{P} P$ is equicontinuous.

On the other hand, if $p=1$, then it is clear that $\mathscr{F} p$ is equicontinuous whenever every point of $J$ is isolated, while if there exists $t_{0} \in \mathbb{T}$ such that $t_{0}$ is not isolated, then we will prove that $\mathscr{F} P$ is not equicontinuous.

Let $S:=1 /(b-a+1)$, let $\delta>0$ be arbitrary and let $s_{\delta} \in\left(t_{0}-\delta, t_{0}+\delta\right) \cap \mathbb{T}$ be such that $s_{\delta} \neq t_{0}$; it is not a loss of generality assuming $s_{\delta}<t_{0}$.

Define $f_{\delta}: J \rightarrow \mathbb{R}$ as

$$
f_{\delta}:= \begin{cases}\frac{S}{t_{0}-s_{\delta}}, & \text { if } t \in\left(\left[s_{\delta}, t_{0}\right) \cap J\right), \\ 0, & \text { if } t \notin\left(\left[s_{\delta}, t_{0}\right) \cap J\right) ;\end{cases}
$$

the fundamental theorem of Calculus asserts that $F_{\delta}: J \rightarrow \mathbb{R}$ given by

$$
F_{\delta}(t):=\int_{[a, t) \cap \mathbb{\pi}} f_{\delta}(s) \Delta s, \quad t \in J
$$

belongs to $\mathscr{F}_{p}$; so that, as

$$
F_{\delta}\left(t_{0}\right)-F_{\delta}\left(s_{\delta}\right)=\int_{\left[s_{\delta}, t_{0}\right) \cap \mathbb{W}} f_{\delta}(s) \Delta s=S,
$$

we conclude that $\mathscr{F} P$ is not equicontinuous.

Therefore, Arzelà-Ascoli theorem establishes our claims.

As a consequence of Proposition 3.6, we achieve the following sufficient condition for strong convergence in $C(J)$.

Corollary 3.8. Let $p \in \overline{\mathbb{R}}$ be such that $p>1$, let $\left\{x_{m}\right\}_{m \in \mathbb{N}} \subset W_{\Delta}^{1, p}(J)$, and let $x \in W_{\Delta}^{1, p}(J)$. If $\left\{x_{m}\right\}_{m \in \mathbb{N}}$ converges weakly in $W_{\Delta}^{1, p}(J)$ to $x$, then $\left\{x_{m}\right\}_{m \in \mathbb{N}}$ converges strongly in $C(J)$ to $x$.

Proof. Suppose $\left\{x_{m}\right\}_{m \in \mathbb{N}}$ converges weakly in $W_{\Delta}^{1, p}(J)$ to $x$; Proposition 3.6 establishes that $\left\{x_{m}\right\}_{m \in \mathbb{N}}$ converges weakly in $C(J)$ to $x$ and so, as $\left\{x_{m}\right\}_{m \in \mathbb{N}}$ is equicontinuous, $\left\{x_{m}\right\}_{m \in \mathbb{N}}$ converges strongly in $C(J)$ to $x$.

Moreover, Proposition 3.6 allows to deduce the following equivalence between the Sobolev's spaces on $J, W_{\Delta}^{1, p}(J)$, and the usual Sobolev's spaces on $(a, b), W^{1, p}((a, b))$.

Corollary 3.9. Suppose that $p \in \overline{\mathbb{R}}$ and $p \geq 1, x: J \rightarrow \mathbb{R}$ and $\bar{x}:[a, b] \rightarrow \mathbb{R}$ is the extension of $x$ to $[a, b]$ defined in (2.13). Then, $x$ belongs to $W_{\Delta}^{1, p}(J)$ if and only if $\bar{x}$ belongs to $W^{1, p}((a, b))$. 
Moreover, there exist two constants $K_{1}, K_{2}>0$ which only depend on $(b-a)$ such that the inequalities

$$
K_{1} \cdot\|\bar{x}\|_{W^{1, p}} \leq\|x\|_{W_{\Delta}^{1, p}} \leq K_{2} \cdot\|\bar{x}\|_{W^{1, p}}
$$

are satisfied for every $x \in W_{\Delta}^{1, p}(J)$ and $p \in \overline{\mathbb{R}}$ with $p \geq 1$.

Proof. Let $\bar{x}, \widetilde{x^{\Delta}}:[a, b] \rightarrow \mathbb{R}$ be the extensions of $x$ and $x^{\Delta}$ to $[a, b]$ defined in (2.13) and (2.9), respectively; it is not difficult to deduce the following equality:

$$
\widetilde{x^{\Delta}}=\bar{x}^{\prime} \quad \text { a.e. on }[a, b] \text {. }
$$

Therefore, Corollaries 2.8 and 2.12 and Proposition 3.6 yield to the result.

As an application of the previous result, we will prove that some properties known for $W^{1, p}((a, b))$ are directly transferred to $W_{\Delta}^{1, p}(J)$; in order to do this, we will use the following result.

Proposition 3.10. If $y:[a, b] \rightarrow \mathbb{R}$ belongs to $W^{1, p}((a, b))$ for some $p \in \overline{\mathbb{R}}$ with $p \geq 1$, then $y_{\mid J}$ belongs to $W_{\Delta}^{1, p}(J)$. Moreover, there exists a constant $T>0$ which only depends on $(b-a)$ such that

$$
\left\|y_{\mid J}\right\|_{W_{\Delta}^{1, p}} \leq T \cdot\|y\|_{W^{1, p}}, \quad \forall y \in W^{1, p}((a, b)), p \in \overline{\mathbb{R}}, p \geq 1 .
$$

Proof. Let $R=\left\{t_{i}\right\}_{i \in I}, I \subset \mathbb{N}$, be the set of all right-scattered points of $\mathbb{T}$, let $I_{J^{o}}=\{i \in I$, $\left.t_{i} \in J^{o}\right\}$ and suppose $y \in W^{1, p}((a, b))$ for some $p \in \overline{\mathbb{R}}$ with $p \geq 1$. The classical fundamental theorem of Calculus allows to assert that

$$
\begin{gathered}
\left(y_{\mid J}\right)^{\Delta}\left(t_{i}\right)=\frac{\int_{\left[t_{i}, \sigma\left(t_{i}\right)\right]} y^{\prime}(s) d s}{\sigma\left(t_{i}\right)-t_{i}}, \quad \text { for every } i \in I_{J^{o}}, \\
\left(y_{\mid J}\right)^{\Delta}=y^{\prime} \quad \text { a.e. on } J^{o} \cap(\mathbb{T} \backslash R) .
\end{gathered}
$$

Therefore, if $p=+\infty$, then it is clear that $y_{\mid J} \in W_{\Delta}^{1, p}(J)$ and (3.28) holds while if $p \in \mathbb{R}$, then, by (2.2), we have that

$$
\left\|\left(y_{\mid J}\right)^{\Delta}\right\|_{L_{\Delta}^{p}}^{p} \leq \int_{J^{o} \cap(\mathbb{T} \backslash R)}\left|y^{\prime}\right|^{p}(s) d s+\sum_{i \in I_{J^{o}}} \int_{\left[t_{i}, \sigma\left(t_{i}\right)\right]}\left|y^{\prime}\right|^{p}(s) d s \leq\|y\|_{W^{1, p}}^{p},
$$

moreover, as we know that

$$
\left\|y_{\mid J}\right\|_{L_{\Delta}^{p}} \leq(b-a)^{1 / p} \cdot\|y\|_{C([a, b])} \leq C \cdot(b-a)^{1 / p} \cdot\|y\|_{W^{1, p}},
$$

for some $C>0$, it turns out that $y_{\mid J} \in W_{\Delta}^{1, p}(J)$ and (3.28) is true.

Next, we deduce some properties in $W_{\Delta}^{1, p}(J)$ from the analogous ones in $W^{1, p}((a, b))$. Corollary 3.11. Let $p \in \overline{\mathbb{R}}$ be such that $p \geq 1$. Then, for every $q \in[1,+\infty)$, the inmersion $W_{\Delta}^{1, p}(J) \hookrightarrow L_{\Delta}^{q}\left(J^{o}\right)$ is compact. 
Proof. Fix $q \in[1,+\infty)$; as a consequence of Proposition 3.7 and the fact that the inmersion $C(J) \hookrightarrow L_{\Delta}^{q}\left(J^{o}\right)$ is continuous, it only remains to prove that $\mathscr{F}_{1}$ is compact in $L_{\Delta}^{q}\left(J^{o}\right)$ whenever $J$ has at least one not isolated point.

Assume the existence of a not isolated point $t_{0} \in J$ and let $\left\{x_{n}\right\}_{n \in \mathbb{N}}$ be a sequence in $\mathscr{F}^{1}$. Corollary 3.9 ensures that $\left\{\bar{x}_{n}\right\}_{n \in \mathbb{N}}$, defined in (2.13), is a bounded sequence in $W^{1,1}((a, b))$ and hence, there exist $\left\{\overline{x_{n_{k}}}\right\}_{k \in \mathbb{N}}$ and $y \in L^{q}([a, b])$ such that $\left\{\overline{x_{n_{k}}}\right\}_{k \in \mathbb{N}}$ converges strongly in $L^{q}([a, b])$ to $y$. By defining $x:=y_{\mid J}$, it is not difficult to prove that $\left\{x_{n_{k}}\right\}_{k \in \mathbb{N}}$ converges strongly in $L_{\Delta}^{q}\left(J^{o}\right)$ to $x$.

Corollary 3.12. The Banach space $W_{\Delta}^{1, p}(J)$ is reflexive for every $p \in(1,+\infty)$ and separable for all $p \in[1,+\infty)$.

Proof. Let $p \in \overline{\mathbb{R}}$ be such that $p \geq 1$. We know, from Corollary 3.9, that the operator $T_{p}$ : $W_{\Delta}^{1, p}(J) \rightarrow W^{1, p}((a, b))$ given for every $x \in W_{\Delta}^{1, p}(J)$ by $T_{p}(x):=\bar{x}$, defined in (2.13), is linear and continuous. It follows from Corollary 3.9 and Proposition 3.10 that $T_{p}\left(W_{\Delta}^{1, p}(J)\right)$ is a closed subspace of $W^{1, p}((a, b))$. Therefore, since $W^{1, p}((a, b))$ is reflexive whenever $p \in(1,+\infty)$ and separable whenever $p \in[1,+\infty), T_{p}\left(W_{\Delta}^{1, p}(J)\right)$ satisfies the same properties.

Corollary 3.13. If $x \in W_{\Delta}^{1, p}(J)$ for some $p \in[1,+\infty)$, then there exists a sequence of infinitely differentiable functions with compact support in $\mathbb{R},\left\{y_{n}\right\}_{n \in \mathbb{N}}$ such that $\left\{y_{n \mid J}\right\}_{n \in \mathbb{N}}$ converges strongly in $W_{\Delta}^{1, p}(J)$ to $x$.

Proof. Corollary 3.9 asserts that $\bar{x}:[a, b] \rightarrow \mathbb{R}$, defined in (2.13), belongs to $W^{1, p}((a, b))$; so that, there exists a sequence $\left\{y_{n}\right\}_{n \in \mathbb{N}}$ of infinitely differentiable functions with compact support in $\mathbb{R}$ such that $\left\{y_{n \mid[a, b]}\right\}_{n \in \mathbb{N}}$ converges to $\bar{x}$ in $W^{1, p}((a, b))$. Hence, our claim follows from equality $\bar{x}_{\mid J}=x$ and Proposition 3.10 .

3.2. The spaces $W_{0, \Delta}^{1, p}(J)$. Corollary 3.13 guarantees the density of the set $C_{r d}^{1}\left(J^{\kappa}\right)$ in $W_{\Delta}^{1, p}(J)$ for every $p \in[1,+\infty)$; however, for an arbitrary bounded time scale it is not true that the set of test functions defined in (3.2), $C_{0, r d}^{1}\left(J^{\kappa}\right)$, is dense in $W_{\Delta}^{1, p}(J)$; this section is devoted to prove some properties concerning the closure of $C_{0, r d}^{1}\left(J^{\kappa}\right)$ in $W_{\Delta}^{1, p}(J)$.

Definition 3.14. Let $p \in \mathbb{R}$ be such that $p \geq 1$, define the set $W_{0, \Delta}^{1, p}(J)$ as the closure of the set $C_{0, r d}^{1}\left(J^{\kappa}\right)$ in $W_{\Delta}^{1, p}(J)$. Denote as $H_{0, \Delta}^{1}(J):=W_{0, \Delta}^{1,2}(J)$.

The spaces $W_{0, \Delta}^{1, p}(J)$ and $H_{0, \Delta}^{1}(J)$ are endowed with the norm induced by $\|\cdot\|_{W_{\Delta}^{1, p} \text {, de- }}$ fined in (3.18), and the inner product induced by $(\cdot, \cdot)_{H_{\Delta}^{1}}$, defined in (3.19), respectively. Since $W_{0, \Delta}^{1, p}(J)$ is closed in $W_{\Delta}^{1, p}(J)$, Theorem 3.5 and Corollary 3.12 ensure that $W_{0, \Delta}^{1, p}(J)$ is a separable Banach space and reflexive whenever $p>1$ and $H_{0, \Delta}^{1}(J)$ is a separable Hilbert space. The space $W_{0, \Delta}^{1, p}(J)$ is characterized in the following result.

Proposition 3.15. Assume $x \in W_{\Delta}^{1, p}(J)$. Then, $x \in W_{0, \Delta}^{1, p}(J)$ if and only if $x(a)=0=x(b)$. 
Proof. Firstly, suppose that $x \in W_{0, \Delta}^{1, p}(J)$, so that there exists a sequence $\left\{x_{n}\right\}_{n \in \mathbb{N}} \subset C_{0, r d}^{1}\left(J^{\kappa}\right)$ such that $\left\{x_{n}\right\}_{n \in \mathbb{N}}$ converges strongly in $W_{\Delta}^{1, p}(J)$ to $x$. Therefore, inequality (3.21) allows to assert that $x(a)=0=x(b)$.

Conversely, assume that $x(a)=0=x(b)$. We know from Corollary 3.9 that $\bar{x}:[a, b] \rightarrow$ $\mathbb{R}$, defined in (2.13), belongs to $W_{0}^{1, p}((a, b))$ and so, there exists a sequence $\left\{y_{n}\right\}_{n \in \mathbb{N}} \subset$ $C_{c}^{1}((a, b))$ which converges strongly in $W^{1, p}((a, b))$ to $\bar{x}$. By defining $x_{n}:=y_{n \mid J}, n \in \mathbb{N}$, one can deduce that $x_{n} \in C_{0, r d}^{1}\left(J^{\kappa}\right)$ for every $n \in \mathbb{N}$ and $\left\{x_{n}\right\}_{n \in \mathbb{N}}$ converges strongly in $W_{\Delta}^{1, p}(J)$ to $x$.

As a straightforward consequence of the previous result, Corollary 3.9, and the characterization of $W_{0}^{1, p}((a, b))$ we obtain the following criterion for belonging to $W_{0, \Delta}^{1, p}(\mathbb{T})$.

Corollary 3.16. Let $p \in \mathbb{R}$ be such that $p \geq 1$, let $x: J \rightarrow \mathbb{R}$, and let $\bar{x}:[a, b] \rightarrow \mathbb{R}$ be the extension of $x$ to $[a, b]$ defined in (2.13). Then, $x \in W_{0, \Delta}^{1, p}(J)$ if and only if $\bar{x} \in W_{0}^{1, p}((a, b))$.

By using Proposition 3.15, we are able to prove the validity of Poincarés inequality.

Proposition 3.17. Let $p \in \mathbb{R}$ be such that $p \geq 1$. Then, there exists a constant $L>0$, only dependent on $(b-a)$, such that

$$
\|x\|_{W_{\Delta}^{1, p}} \leq L \cdot\left\|x^{\Delta}\right\|_{L_{\Delta}^{p}}, \quad \forall x \in W_{0, \Delta}^{1, p}(J)
$$

that is, in $W_{0, \Delta}^{1, p}(J)$, the norm defined for every $x \in W_{0, \Delta}^{1, p}(J)$ as $\left\|x^{\Delta}\right\|_{L_{\Delta}^{p}}$ is equivalent to the norm $\|\cdot\|_{W_{\Delta}^{1, p} \text {. }}$

Proof. Choose $x \in W_{0, \Delta}^{1, p}(J)$; the fundamental theorem of Calculus and Proposition 3.15 allow to assert that the following inequality

$$
|x(t)|=\left|x(a)+\int_{[a, t) \cap \mathbb{\pi}} x^{\Delta}(s) \Delta s\right|=\left|\int_{[a, t) \cap \mathbb{\pi}} x^{\Delta}(s) \Delta s\right| \leq\left\|x^{\Delta}\right\|_{L_{\Delta}^{1}}
$$

is valid for every $t \in \mathbb{T}$. Thus, (3.32) follows from Hölder's inequality.

Remark 3.18. One can check that the function defined for every $x, y \in H_{0, \Delta}^{1}(J)$ as $\left(x^{\Delta}, y^{\Delta}\right)_{L_{\Delta}^{2}}$ is an inner product in $H_{0, \Delta}^{1}(J)$ and its associated norm is equivalent to the norm associated to $(\cdot, \cdot)_{H_{\Delta}^{1}}$.

\section{Generalization to order $n \geq 2$}

The aim of this section is to define recursively the $n$ th-order Sobolev's spaces on $J$ for $n \geq 2, W_{\Delta}^{n, p}(J)$, which consist in the $\Delta$-antiderivatives of functions in $W_{\Delta}^{n-1, p}\left(J^{\kappa}\right)$.

Definition 4.1. Let $n \in \mathbb{N}, n \geq 2$, let $p \in \overline{\mathbb{R}}, p \geq 1$, and let $u: J \rightarrow \overline{\mathbb{R}}$. Say that $u$ belongs to $W_{\Delta}^{n, p}(J)$ if and only if $u \in W_{\Delta}^{n-1, p}(J)$ and there exists $g_{1}: J^{\kappa} \rightarrow \mathbb{R}$ such that $g_{1} \in W_{\Delta}^{n-1, p}\left(J^{\kappa}\right)$ and

$$
\int_{J^{o}}\left(u \cdot \varphi^{\Delta}\right)(s) \Delta s=-\int_{J^{o}}\left(g_{1} \cdot \varphi^{\sigma}\right)(s) \Delta s, \quad \forall \varphi \in C_{0, r d}^{1}\left(J^{\kappa}\right) .
$$


It is easy to prove the following characterization of the set $W_{\Delta}^{n, p}(J)$.

Proposition 4.2. Suppose that $u: J \rightarrow \overline{\mathbb{R}}$ is such that $u \in L_{\Delta}^{p}\left(J^{o}\right)$, then $u \in W_{\Delta}^{n, p}(J)$ if and only if there exist $g_{j}: J^{\kappa^{j}} \rightarrow \overline{\mathbb{R}}, j \in\{1, \ldots, n\}$, such that $g_{j} \in L_{\Delta}^{p}\left(\left(J^{\kappa^{j-1}}\right)^{o}\right)$,

$$
\int_{J^{o}}\left(u \cdot \varphi^{\Delta}\right)(s) \Delta s=-\int_{J^{o}}\left(g_{1} \cdot \varphi^{\sigma}\right)(s) \Delta s, \quad \forall \varphi \in C_{0, r d}^{1}\left(J^{\kappa}\right),
$$

and for all $j \in\{2, \ldots, n\}$,

$$
\int_{\left(J^{k^{j-1}}\right)^{o}}\left(g_{j-1} \cdot \varphi^{\Delta}\right)(s) \Delta s=-\int_{\left(J^{k^{j-1}}\right)^{o}}\left(g_{j} \cdot \varphi^{\sigma}\right)(s) \Delta s, \quad \forall \varphi \in C_{0, r d}^{1}\left(J^{k^{j}}\right),
$$

with

$$
C_{0, r d}^{1}\left(J^{\kappa^{j}}\right):=\left\{f: J^{\kappa^{j-1}} \longrightarrow \mathbb{R}: f \in C_{r d}^{1}\left(J^{\kappa^{j}}\right), f(a)=0=f\left(\rho^{j-1}(b)\right)\right\}
$$

and $C_{r d}^{1}\left(J^{\kappa^{j}}\right)$ is the set of all continuous functions on $J^{\kappa^{j-1}}$ such that they are $\Delta$-differentiable on $J^{\kappa^{j}}$ and their $\Delta$-derivatives are $r d$-continuous on $J^{\kappa^{j}}$.

The integration by parts formula for absolutely continuous functions on closed subintervals of $\mathbb{T}$ establishes that the relation

$$
V_{\Delta}^{n, p}(J):=\left\{x \in A C^{n-1}(J): x^{\Delta^{n}} \in L_{\Delta}^{p}\left(\left(J^{\kappa^{n-1}}\right)^{o}\right)\right\} \subset W_{\Delta}^{n, p}(J)
$$

is true for every $p \in \overline{\mathbb{R}}$ with $p \geq 1$; moreover, both sets are, as class of functions, equivalent as one can check in the following result.

Theorem 4.3. Suppose that $u \in W_{\Delta}^{n, p}(J)$ for some $n \in \mathbb{N}$ with $n \geq 2, p \in \mathbb{R}$ with $p \geq 1$ and that (4.1) holds for $g_{1} \in L_{\Delta}^{p}\left(J^{o}\right)$. Then, there exists a unique function $x \in V_{\Delta}^{n, p}(J)$ such that

$$
x=u \quad \Delta \text {-a.e. on } J^{o}, \quad x^{\Delta^{j}}=g_{j} \quad \Delta \text {-a.e. on }\left(J^{k^{j-1}}\right)^{o}, 1 \leq j \leq n,
$$

where $J^{\kappa^{0}}=J$ and $g_{j}: J^{\kappa^{j}} \rightarrow \overline{\mathbb{R}}, 1 \leq j \leq n$, are given in Proposition 4.2.

Inductively, one can prove that the set $W_{\Delta}^{n, p}(J)$ is endowed with the structure of Banach space.

Theorem 4.4. Assume $n \in \mathbb{N}, n \geq 2, p \in \overline{\mathbb{R}}$ and $p \geq 1$. The set $W_{\Delta}^{n, p}(J)$ is a Banach space together with the norm defined for every $x \in W_{\Delta}^{n, p}(J)$ as

$$
\|x\|_{W_{\Delta}^{n, p}}:=\sum_{j=0}^{n}\left\|x^{\Delta^{j}}\right\|_{L_{\Delta}^{p}},
$$

where $x^{\Delta^{0}}=x$. Furthermore, the set $H_{\Delta}^{n}(J):=W_{\Delta}^{n, 2}(J)$ is a Hilbert space together with the inner product given for every $(x, y) \in H_{\Delta}^{n}(J) \times H_{\Delta}^{n}(J)$ by

$$
(x, y)_{H_{\Delta}^{n}}:=\sum_{j=0}^{n}\left(x^{\Delta^{j}}, y^{\Delta^{j}}\right)_{L_{\Delta}^{2}} .
$$


Properties proved for the spaces $W_{\Delta}^{1, p}(J)$ can be derived for the spaces $W_{\Delta}^{n, p}(J)$; for instance, we have the following.

Proposition 4.5. The immersion $W_{\Delta}^{n, p}(J) \hookrightarrow C^{n-1}\left(J^{\kappa^{n-1}}\right)$ is continuous; where $C^{n-1}\left(J^{\kappa^{n-1}}\right)$ is the set of all functions defined on $J$ with $n-1$ continuous $\Delta$-derivatives on $J^{\kappa^{j}}, 1 \leq j \leq$ $n-1$.

Finally, by extending, whenever it is necessary, the function $x^{\Delta^{n-1}}$ to $J$ as

$$
x^{\Delta^{n-1}}\left(\rho^{j}(b)\right)=x^{\Delta^{n-1}}\left(\rho^{n-1}(b)\right) \quad \forall j \in\{0, \ldots, n-2\},
$$

with $\rho^{0}(b)=b$, one can prove inductively the following relation between the Banach spaces $W_{\Delta}^{n, p}(J)$ and $W^{n, p}((a, b))$.

Theorem 4.6. Let $n \in \mathbb{N}, n \geq 2$, let $x: J \rightarrow \mathbb{R}$ be such that $x \in C^{n-1}\left(J^{\kappa^{n-1}}\right)$.

Then, $x \in W_{\Delta}^{n, p}(J)$ if and only if the function $y:[a, b] \rightarrow \mathbb{R}$ defined for every $t \in[a, b]$ as

$$
y(t):=\sum_{j=0}^{n-2} x^{\Delta^{j}}(a) \frac{(t-a)^{j}}{j !}+\int_{A_{t}} \overline{x^{\Delta^{n-1}}}\left(s_{n-1}\right) d s_{n-1} \cdots d s_{1}
$$

belongs to $W^{n, p}((a, b))$, where $\overline{x^{\Delta^{n-1}}}:[a, b] \rightarrow \mathbb{R}$ is the extension of $x^{\Delta^{n-1}}: J^{\kappa^{n-1}} \rightarrow \mathbb{R}$ defined in (2.13) and

$$
A_{t}:=\left\{\left(s_{1}, \ldots, s_{n-1}\right) \in[a, b]^{n-1}: s_{n-1}<\cdots<s_{1}<t\right\} .
$$

Moreover, the following equalities

$$
y^{n}=x^{\Delta^{n}} \quad \Delta \text {-a.e. on } J^{\kappa^{n}}, \quad y^{n-1}=x^{\Delta^{n-1}} \quad \text { on } J^{\kappa^{n-1}}
$$

hold.

\section{Acknowlegments}

This research is partially supported by D.G.I. and F.E.D.E.R. project MTM 2004-06652C03-01, and by Xunta of Galicia and F.E.D.E.R. project PGIDIT05PXIC20702PN, Spain.

\section{References}

[1] M. Bohner and A. Peterson (eds.), Advances in Dynamic Equations on Time Scales, Birkhäuser Boston, Massachusetts, 2003.

[2] H. Brezis, Analyse Fonctionnelle: Thèorie et Applications, Masson, Paris, 1996.

[3] A. Cabada and D. R. Vivero, Criterions for absolute continuity on time scales, Journal of Difference Equations and Applications 11 (2005), no. 11, 1013-1028.

[4] __ Expression of the Lebesgue $\Delta$-integral on time scales as a usual Lebesgue integral. Application to the calculus of $\Delta$-antiderivatives, Journal of Mathematical Analysis and Applications 43 (2006), 194-207.

[5] G. Sh. Guseinov, Integration on time scales, Journal of Mathematical Analysis and Applications 285 (2003), no. 1, 107-127. 


\section{Basic properties of Sobolev's spaces on time scales}

[6] E. Hewitt and K. Stromberg, Real and Abstract Analysis. A Modern Treatment of the Theory of Functions of a Real Variable, 3rd ed., Graduate Texts in Mathematics, no. 25, Springer, New York, 1975.

[7] W. Rudin, Real and Complex Analysis, 1st ed., McGraw-Hill, New York, 1966.

[8] _ Real and Complex Analysis, 3rd ed., McGraw-Hill, New York, 1987.

Ravi P. Agarwal: Department of Mathematical Sciences, Florida Institute of Technology, Melbourne, FL 32901, USA

E-mail address: agarwal@fit.edu

Victoria Otero-Espinar: Departamento de Análise Matemática, Facultade de Matemáticas, Universidade de Santiago de Compostela, 15782 Santiago de Compostela, Galicia, Spain E-mail address: vivioe@usc.es

Kanishka Perera: Department of Mathematical Sciences, Florida Institute of Technology, Melbourne, FL 32901, USA

E-mail address: kperera@fit.edu

Dolores R. Vivero: Departamento de Análise Matemática, Facultade de Matemáticas, Universidade de Santiago de Compostela, 15782 Santiago de Compostela, Galicia, Spain E-mail address: lolirv@usc.es 